\title{
The EGFR-rearranged adenocarcinoma is associated with a high rate of venous thromboembolism
}

\author{
Jing Wang, Bin Hu, Tong Li, Jinbai Miao, Wenqian Zhang, Shuo Chen, Yixin Sun, Songping Cui, Hui Li \\ Department of Thoracic Surgery, Beijing Chaoyang Hospital, Capital Medical University, Beijing 100020, China \\ Contributions: (I) Conception and design: J Wang, H Li; (II) Administrative support: H Li; (III) Provision of study materials or patients: J Wang, H \\ Li; (IV) Collection and assembly of data: J Wang, Y Sun, S Cui; (V) Data analysis and interpretation: J Wang; (VI) Manuscript writing: All authors; (VII) \\ Final approval of manuscript: All authors. \\ Correspondence to: Professor Hui Li. Beijing Chaoyang Hospital, Capital Medical University, Beijing 100020, China. Email: huilee@vip.sina.com.
}

Background: The purpose of this study was to investigate the incidence of venous thromboembolism (VTE) in epidermal growth factor receptor (EGFR) mutations patients with lung adenocarcinoma, to provide clinical basis for the perioperative prevention and treatment of VTE in patients with lung cancer.

Methods: This study included patients with invasive lung adenocarcinoma confirmed by pathology from July 2016 to March 2018 after surgical pulmonectomy in Thoracic Surgery Department of Beijing Chaoyang Hospital. All enrolled patients were tested for relevant gene mutations. All patients were classified as adenocarcinoma subtypes by the 2011 International Association for the Study of Lung Cancer (IASLC), American Thoracic Society (ATS) and European Respiratory Society (ERS). Patients were divided into the VTE group and the control group according to whether VTE occurred postoperatively. Baseline data, gene test results, operative data and tumor pathology data between the two groups were compared.

Results: According to the inclusion criteria, a total of 323 patients underwent lung cancer surgery were analyzed in this study, including 148 males and 175 females, aged from 25 to 82 years old. Postoperative VTE occurred in 33 patients, with an incidence of $10.2 \%$. Compared the baseline data, there were significant differences in age and BMI between the two groups, but no significant differences in other indicators. Comparing the results of postoperative genetic tests, the cases of exon 18, 19, 20, 21, 30, 31 mutation, exon 18 and 20 mixed mutation and exon 20 and 21 mixed mutation were 5, 42, 6, 57, 1, 3, 1 and 1. The total EGFR mutation rate in the enrolled patients was 36.2\% (117/323). Among them, the proportion of EGFR mutation in the VTE group was significantly higher than that in the non-VTE group (60.6\% vs. 33.4\%, $\mathrm{P}=0.002)$. Exon mutations in specific regions and mixed region of EGFR were not statistically significant between them; there was no statistical difference in the concomitant KRAS and ALK gene mutations between them. Comparing the pathological conditions, the proportion of acinar dominant lung adenocarcinoma in the VTE group was higher than that in the non-VTE group (57.6\% vs. 30.7\%, $\mathrm{P}=0.002$ ); other histologic subtypes showed no statistical difference. The D-dimer difference before and 1 day after surgery, preoperative FEV1, surgical method, duration of surgery and blood loss were statistically significant differences between the two groups. The results of univariate analysis showed that there were significant differences between the VTE group and the control group in proportion of EGFR mutant lung adenocarcinoma, age, BMI, D-dimer difference before and 1 day after surgery, preoperative FEV1, surgical method, duration of surgery, blood loss and proportion of acinar dominant lung adenocarcinoma $(\mathrm{P}<0.05)$. However, VTE was not significantly correlated with gender, $A L K$ or $K R A S$ gene mutation and other factors. Multi-factor logistics regression analysis shows that Patients with EGFR gene mutation infiltrating lung adenocarcinoma, acinar dominant lung adenocarcinoma, FEV1 and difference of D-dimer (d1-pre) are independent risk factors for postoperative lung cancer complicated with VTE.

Conclusions: The incidence of VTE was $10.2 \%$ in patients with invasive lung adenocarcinoma without prophylactic anticoagulant therapy. EGFR gene mutation is an independent risk factor for postoperative VTE in lung cancer, and the incidence of VTE in adenocarcinoma with alveolar predominance is the 
highest. Other independent risk factors included the difference of D-dimer (d1-pre) and preoperative FEV1.

Keywords: Venous thromboembolism (VTE); epidermal growth factor receptor mutant lung adenocarcinoma; adenocarcinoma with alveolar predominance; thoracic surgery

Submitted Sep 08, 2019. Accepted for publication Nov 15, 2019.

doi: 10.21037/atm.2019.12.24

View this article at: http://dx.doi.org/10.21037/atm.2019.12.24

\section{Introduction}

Venous thromboembolism (VTE) is a common complication of patients with tumors, and is one of the important causes of death of patients with tumors. VTE mainly includes deep venous thrombosis (DVT) and pulmonary thromboembolism (PTE), etc. The overall risk of VTE in cancer patients is 7-8 times higher than that in the general population (1). Patients hospitalized and receiving active anti-tumor therapy are the high-risk groups for VTE. Lung cancer is one of the most common malignant tumors in patients with VTE, and the occurrence of VTE is one of the important causes of death in patients with lung cancer. In our previous study, the incidence of VTE after benign lung tumors was $7.5 \%$, and the incidence of malignant tumors was $16.4 \%$ (2), which further confirmed this view. Studies have found that some nonsmall cell lung cancer (NSCLC) cells have high expression of epidermal growth factor receptor (EGFR), and some NSCLC cells are sensitive to EGFR-TKI drugs, which all suggest that some NSCLC is the result of abnormal expression of EGFR (3-5). The EGFR, a member of the ErbB receptor tyrosine kinase family, is activated by EGF or heparin binding EGF (HB-EGF) in an autocrine or paracrine manner. After ligand binding, EGFR forms homo- or heterodimers with all four family members and activates various signaling pathways that affect vascular cell differentiation and matrix homeostasis. EGFR can also be bound to specific receptors by vasoactive agents such as angiotensin, $\alpha 1, \beta$-adrenergic receptor agonists, thrombin, etc., leading to reverse transcription that acts on vascular endothelial cells (2). NSCLC accounts for $75-85 \%$ of the total cases of lung cancer, among which adenocarcinoma is the main type. In 2011, International Association for the Study of Lung Cancer (IASLC), American Thoracic Society (ATS) and European Respiratory Society (ERS) jointly initiated an international multidisciplinary approach to the classification of lung adenocarcinoma (6). The new classification method of lung adenocarcinoma provides a uniform terminology and diagnostic criteria, and cancels the classification of bronchiolar alveolar carcinoma. Four new names have been added: adenocarcinoma in situ, microinfiltrating adenocarcinoma, infiltrating adenocarcinoma, and infiltrating mucinous adenocarcinoma. Among them, infiltrating adenocarcinoma includes adherent, acinar, papillary, micropapillary, solid, mucinous, fetal, colloidal, and intestinal adenocarcinoma. Currently, there are few relatively studies on the incidence of VTE in patients with invasive lung adenocarcinoma with specific molecular subtypes. The purpose of this study is to observe the clinicopathological data of VTE patients after lung cancer surgery, in order to investigate the incidence of VTE in patients with EGFR mutated lung adenocarcinoma and the related risk factors affecting the occurrence of VTE in patients with lung cancer, so as to provide clinical basis for the perioperative prevention and treatment of VTE in patients with lung cancer.

\section{Methods}

\section{Ethics approval and consent to participate:}

The study was approved by the Ethics Committee of Beijing Chaoyang Hospital (No.2019-D.-221-1). The participants provided written informed consent to participate in this study.

\section{Study object}

This study was a single-center study, with cases of lung cancer undergoing surgical treatment in the department of thoracic surgery of our hospital from July 2016 to March 2018 as the study object. The authors are accountable for all aspects of the work in ensuring that questions related to the accuracy or integrity of any part of the work are appropriately investigated and resolved.

Inclusion criteria: (I) patients with primary infiltrating lung adenocarcinoma; (II) surgical treatment has been 


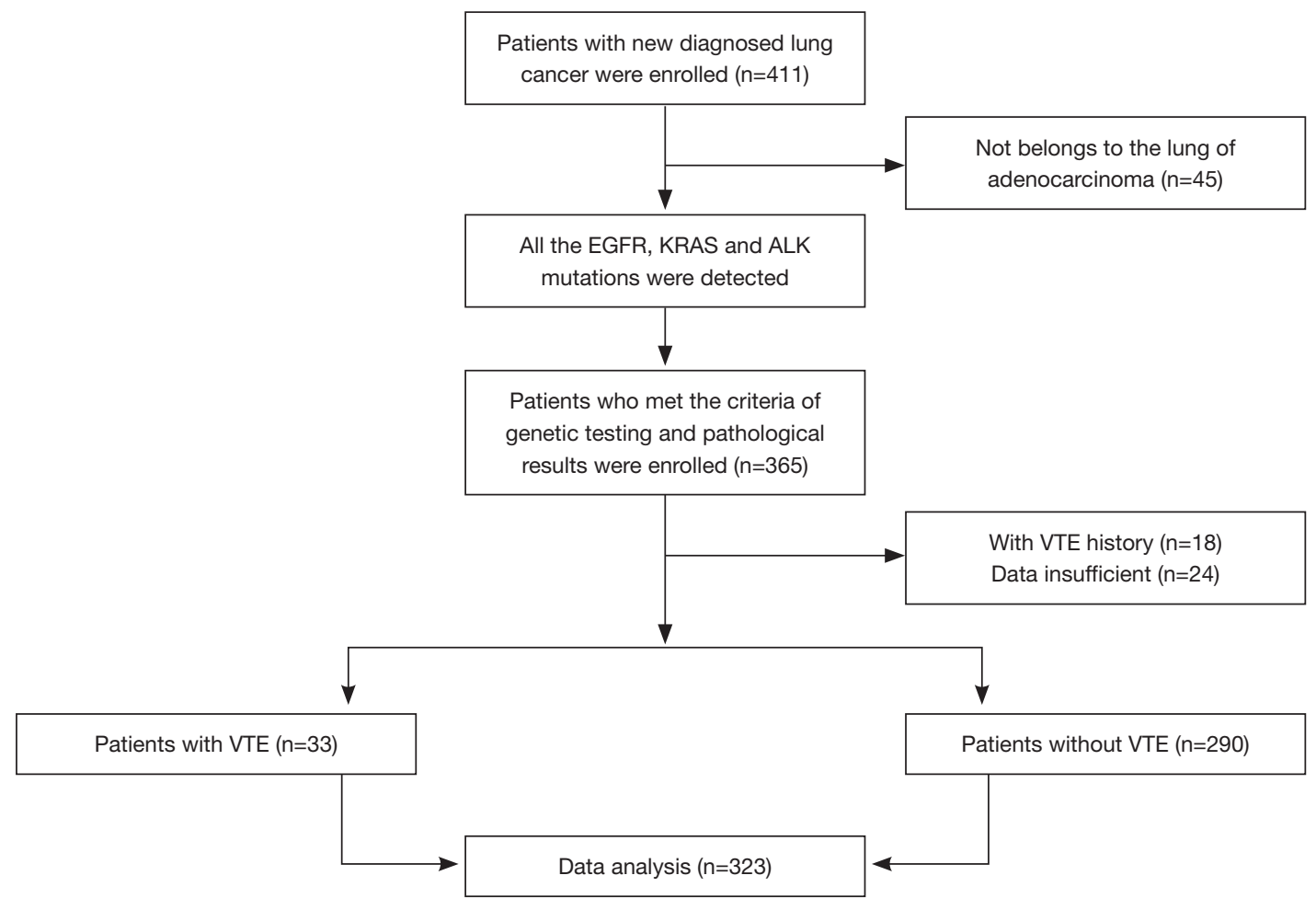

Figure 1 Screening flow chart.

carried out; (III) ultrasound examination of lower limb veins before and after surgery; (IV) complete pathological results of genetic testing were obtained after the operation.

Exclusion criteria: (I) metastatic lung cancer; (II) deep vein thrombosis (DVT) was identified by preoperative low limb ultrasound; (III) no lower extremity Doppler ultrasonography was performed; (IV) other diseases of the blood system; (V) anticoagulation therapy was applied for other diseases before and after operation. Below is our grouping flowchart (Figure 1).

\section{Data collection}

\section{Collection of general data}

Data of all subjects were collected in a unified Excel form, including basic information of patients (including age, gender, combined diseases, smoking history, BMI, etc.); laboratory examination (including $\mathrm{D}$-dimer values before surgery and after surgery at days 1, 3 and 5, patient blood type, etc.); preoperative pulmonary function examination (including FEV1, etc.); genetic test results (with or without genetic mutation, mutant gene type, pure mutation or mixed mutation, etc.); operation information (operation mode, operation duration, blood loss, etc.); pathological data of tumor (tumor classification, mixed subtype, degree of differentiation, vascular invasion, vascular thrombus, etc.).

\section{VTE related tests}

Caprini risk stratification was carried out in all patients, with scores $\leq 4$ points for VTE low-risk, 5-8 points for medium-risk and $\geq 9$ points for high-risk. All the enrolled patients received doppler ultrasound of lower limbs before and after the operation to determine whether there was DVT or not. If there are any of the following problems, CT pulmonary angiogram (CTPA) should be performed to determine whether there is a new pulmonary embolism (PE): (I) PTE symptoms (chest pain, hemoptysis or unexplained hypoxemia and dyspnea); (II) Caprini score $\geq 9$ points; (III) new DVT occurred after surgery.

\section{Diagnostic basis and criteria}

The diagnosis of lung cancer is based on the pathological results of tumor. All patients with lung adenocarcinoma are classified into subtypes of lung adenocarcinoma according to the classification method proposed by IASLC/ATS/ERS 
Table 1 Analysis of baseline information of lung cancer patients

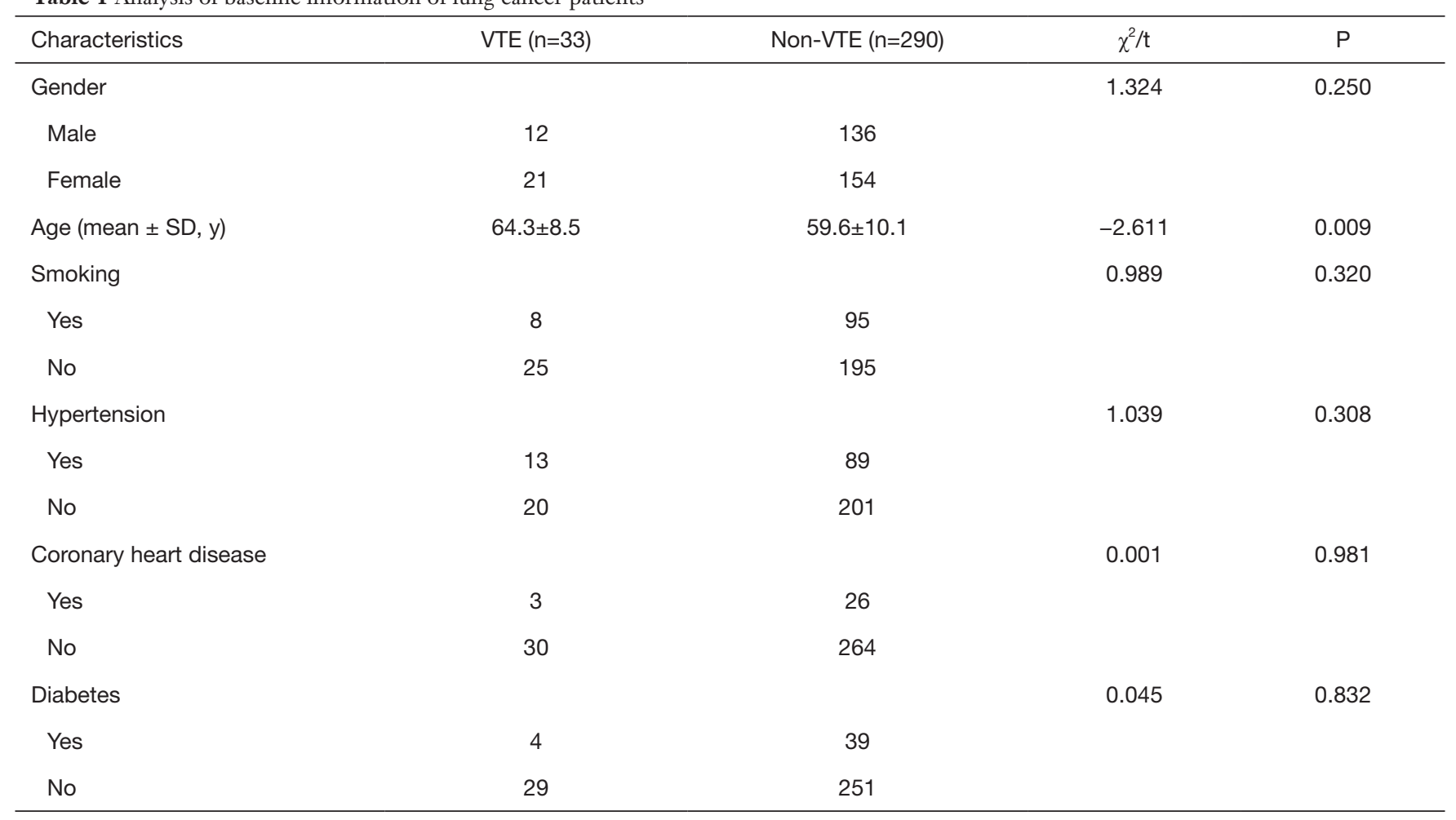

VTE, venous thromboembolism.

in 2011. DVT was diagnosed by color doppler ultrasound. PTE was diagnosed by CT pulmonary angiography. Postoperative VTE positive was defined as no VTE before surgery, and DVT (including intramural venous thrombosis) was diagnosed after surgery by ultrasound of lower extremity or PE by CTPA.

\section{Statistical methods}

The measurement data of normal distribution were expressed as Mean \pm standard deviation (mean \pm SD), and the $t$-test was used for comparison between groups. Measurement data that do not obey normal distribution are represented by $M(Q R)$, and non-parametric test is adopted for comparison between groups. The counters were measured by ratio and rate, and compared by $\chi^{2}$ test. Variables with statistical significance from univariate analysis results were included in multivariate Logistic regression analysis. All data were statistically analyzed using SPSS22.0 software package, and $\mathrm{P}<0.05$ was considered statistically significant.

\section{Results}

\section{Baseline data analysis}

From July 2016 to March 2018, a total of 323 patients with invasive lung adenocarcinoma were enrolled, among which 33 patients were diagnosed with VTE postoperatively (VTE group), including 29 patients with DVT, 1 patient with PTE, and 3 patients with DVT combined with PE, 290 patients with non-VTE (control group). The age of the VTE group was significantly higher than that of the control group $(64.3 \pm 8.5$ vs. $59.6 \pm 10.1, \mathrm{P}=0.009)$. BMI of the VTE group was higher than that of the control group $(25.2 \pm 4.0$ vs. 23.9 $\pm 3.1, \mathrm{P}=0.035)$. There was no statistical difference between the VTE group and the control group in terms of gender, smoking, complications, etc. (Table 1).

\section{Analysis of preoperative auxiliary examination data}

All patients underwent blood group test, coagulation test, lung function test and other tests before the surgery. Among them, the preoperative D-dimer level in the VTE 
Table 2 Analysis of preoperative auxiliary examination of lung cancer patients

\begin{tabular}{|c|c|c|c|c|}
\hline Variables & VTE $(n=33)$ & Non-VTE $(n=290)$ & $\chi^{2} / \mathrm{t}$ & $\mathrm{P}$ \\
\hline D-dimer (before operation) & $0.89 \pm 2.3$ & $0.42 \pm 0.6$ & -2.086 & 0.050 \\
\hline D-dimer (the first day after operation) & $2.55 \pm 2.8$ & $1.20 \pm 1.3$ & -4.799 & 0.001 \\
\hline Blood type & & & 1.084 & 0.781 \\
\hline Blood type (B) & 14 & 98 & & \\
\hline Blood type (AB) & 3 & 32 & & \\
\hline Blood type (O) & 9 & 83 & & \\
\hline FEV1 & $2.18 \pm 0.5$ & $2.51 \pm 0.6$ & -1.726 & 0.002 \\
\hline
\end{tabular}

VTE, venous thromboembolism; BMI, body mass index; FEV1, forced expiratory volume-one second.

group was higher than that in the control group $(0.89 \pm 2.3$ vs. $0.42 \pm 0.6)$, but there was no statistical difference $(\mathrm{P}=0.05)$. The increase of D-dimer on the first day after operation in the VTE group was significantly higher than that in the control group $(2.55 \pm 2.8$ vs. $1.2 \pm 1.3)$, which was statistically significant $(\mathrm{P}=0.001)$. Lung function examination: FEV1 in the VTE group was lower than that in the control group $(2.18 \pm 0.5$ vs. $2.51 \pm 0.6)$, with statistical difference $(\mathrm{P}=0.002)$. Blood test was no significant difference between the VTE group and the control group (Table 2).

\section{Analysis of surgical factors}

Sixty-two patients $(19.2 \%)$ underwent thoracotomy and 261 patients $(80.8 \%)$ underwent VATS. The incidence of postoperative VTE in patients undergoing thoracotomy was higher than that in patients undergoing VATS surgery, with statistically significant difference $(\mathrm{P}=0.030)$. The operation duration and blood loss in the VTE group were greater than those in the control group, and there were statistically significant differences $(\mathrm{P}=0.002, \mathrm{P}=0.002)$. The length of postoperative hospitalization in the VTE group was longer than that in the control group, but there was no statistical difference $(\mathrm{P}=0.174)$ (Table 3).

\section{Analysis of gene test results}

The proportion of EGFR mutation in the VTE group was significantly higher than that in the non-VTE group $(60.6 \%$ vs. $33.4 \%, \mathrm{P}=0.002)$, with statistical difference $(\mathrm{P}=0.002)$. There was no statistical difference between the VTE group and the control group in KRAS and $A L K$ gene mutations (Table 4), the cases of exon 18, 19, 20, 21, 30, 31 mutation, exon 18 and 20 mixed mutation and exon 20 and 21 mixed mutation were $5,42,6,57,1,3,1$ and 1 . There was no statistically significant increase in the incidence of exon mutation and VTE in specific regions (Table 4).

\section{Analysis of pathological correlation factors}

Analysis of postoperative pathological correlation factors in this group showed that there were differences in the incidence of VTE in different pathological subtypes of infiltrating lung adenocarcinoma. VTE occurred in 19 cases $(57.6 \%)$ of acinar lung adenocarcinoma, 8 cases (24.2\%) of mural lung adenocarcinoma, 4 cases $(12.1 \%)$ of papillary lung adenocarcinoma, 2 cases (6.1\%) of solid lung adenocarcinoma, and 0 cases of micropapillary lung adenocarcinoma. Among them, the incidence of VTE in acinar lung adenocarcinoma was higher than that in nonacinar lung adenocarcinoma, and there was a statistical difference compared with other subtypes $(\mathrm{P}=0.002)$. No significant differences were observed in vascular infiltration and vascular embolism (Table 5).

\section{Multivariate logistic regression analysis}

The results showed that the difference between preoperative FEV1 and D-dimer on the first day after surgery and preoperative values of lung adenocarcinoma patients with EGFR gene mutation and lung adenocarcinoma dominated by acinar before surgery was an independent risk factor 
Table 3 Analysis of surgical related factors of lung cancer patients

\begin{tabular}{lccc}
\hline Variables & VTE $(\mathrm{n}=33)$ & Non-VTE $(\mathrm{n}=290)$ & $\chi^{2} / \mathrm{t}$ \\
\hline Surgical procedure & 11 & 51 & 0.030 \\
Open thoracotomy & 22 & 239 & 0.002 \\
VATS & $198.6 \pm 5.6$ & $162.7 \pm 5.2$ & -3.065 \\
Length of surgery & $277.2 \pm 157.3$ & $161.8 \pm 44.2$ & -3.007 \\
Bleeding & $7.5 \pm 3.3$ & $6.7 \pm 2.9$ & -1.364 \\
Length of staying & & & 1.240 \\
Vascular invasion & 12 & 135 & 0.174 \\
Yes & 21 & 155 & 0.256 \\
No & 9 & 99 & 0.627 \\
Vascular tumor thrombus & 24 & 191 & 0.428 \\
Yes & 9 & & \\
No & & & \\
\hline
\end{tabular}

VTE, venous thromboembolism; VATS, video-assisted thoracoscopic surgery.

for lung adenocarcinoma with VTE after surgery $(\mathrm{P}<0.05)$ (Table 6).

\section{Conclusions}

VTE is one of the common complications after surgery, and is also an important factor affecting the prognosis of patients (7-9). It is associated with reduced survival rates, substantial waste of medical costs, and high recurrence rates. Although some independent risk factors for VTE and predictors of VTE recurrence have been identified, and effective primary and secondary prevention measures exist, the incidence of VTE appears to be fairly stable, or even increasing. First of all, tumor formation is the result of infinite proliferation of cells, involving the changes of many original oncogenes and tumor suppressor genes, as well as the proliferation ability of tumor cells and the ability to promote angiogenesis and obtain nutrients. Tumor cells can directly express tissue factor ( $\mathrm{TF}$ ) to activate coagulation, and can also act on vascular endothelial cells, fibroblasts and macrophages by secreting IL-1, IL-6, tumor necrosis factor, etc., to make it up-regulate the expression of TFs, regulate the protein $\mathrm{C}$ pathway, activate platelets, indirectly activate coagulation, and promote thrombosis. EGFR is a transmembrane glycoprotein receptor, belonging to the Erb2 family, which has tyrosine kinase activity and plays an important role in promoting cell proliferation and growth. Many epithelial tumors are caused by its abnormal expression. The mechanism of VTE in lung cancer patients is particular complex, EGFR is a transmembrane glycoprotein receptor, belonging to Erb2 family, which has tyrosine kinase activity and plays an important role in promoting cell proliferation and growth. Many epithelial tumors are caused by its abnormal expression. As previously mentioned, the occurrence of some NSCLC is the result of abnormal expression of EGFR. The American association of pathology and IASLC recently published recommendations for molecular testing of EGFR mutations in lung cancer. It states that all patients with advanced lung adenocarcinoma, regardless of gender, race, history of smoking, or other clinical risk factors, should be tested for the EGFR gene. As the number of tumor cells increases and the size of the tumor increases, the demand for oxygen and nutrients will also increase. Tumors must have a strong ability to promote angiogenesis in order to meet this need. A variety of mechanisms lead to the imbalance of the fibrinolyticanticoagulant system in patients. Cancer cells and their metabolites leave patients with hypercoagulable blood (10). Our previous study confirmed that lung cancer is a highrisk factor for VTE (2). However, the present study has some limitations, including the lack of retrospective study on patients with invasive lung adenocarcinoma with this molecular subtype. Therefore, it is important to determine the incidence of VTE in patients with EGFR mutated lung 
Table 4 Analysis of genetic test results of lung cancer patients

\begin{tabular}{|c|c|c|c|}
\hline Variables & VTE $(n=33)$ & Non-VTE $(\mathrm{n}=290)$ & $P$ \\
\hline EGFR & & & 0.002 \\
\hline Mutation & $20(60.6 \%)$ & 97 (33.4\%) & \\
\hline Wild & $13(39.4 \%)$ & $193(66.6 \%)$ & \\
\hline KRAS & & & 0.352 \\
\hline Mutation & 3 & 15 & \\
\hline Wild & 30 & 275 & \\
\hline ALK & & & 0.299 \\
\hline Mutation & 2 & 8 & \\
\hline Wild & 31 & 282 & \\
\hline \multicolumn{4}{|l|}{ EGFR exon } \\
\hline Exon 18 & & & 0.465 \\
\hline Yes & 1 & 4 & \\
\hline No & 32 & 287 & \\
\hline Exon 19 & & & 0.699 \\
\hline Yes & 5 & 37 & \\
\hline No & 28 & 253 & \\
\hline Exon 20 & & & 0.059 \\
\hline Yes & 2 & 4 & \\
\hline No & 31 & 286 & \\
\hline Exon 21 & & & 0.932 \\
\hline Yes & 6 & 51 & \\
\hline No & 27 & 239 & \\
\hline Exon 30 & & & 0.833 \\
\hline Yes & 1 & 0 & \\
\hline No & 32 & 290 & \\
\hline Exon 31 & & & 0.785 \\
\hline Yes & 2 & 1 & \\
\hline No & 31 & 289 & \\
\hline Exon $18+20$ & & & 0.735 \\
\hline Yes & 0 & 1 & \\
\hline No & 33 & 289 & \\
\hline Exon $20+21$ & & & 0.735 \\
\hline Yes & 0 & 1 & \\
\hline No & 33 & 289 & \\
\hline
\end{tabular}

VTE, venous thromboembolism.
Table 5 Analysis of histologic subtype of lung adenocarcinoma patients

\begin{tabular}{|c|c|c|c|}
\hline Histologic subtype & VTE $(n=33)$ & Non-VTE $(\mathrm{n}=290)$ & $\mathrm{P}$ \\
\hline Acinar & & & 0.002 \\
\hline Yes & 19 & 89 & \\
\hline No & 14 & 201 & \\
\hline Papillary & & & 0.226 \\
\hline Yes & 4 & 61 & \\
\hline No & 29 & 229 & \\
\hline Solid & & & 0.098 \\
\hline Yes & 2 & 50 & \\
\hline No & 31 & 240 & \\
\hline Lepidic & & & 0.444 \\
\hline Yes & 8 & 89 & \\
\hline No & 25 & 201 & \\
\hline Micropapillary & & & 0.735 \\
\hline Yes & 0 & 1 & \\
\hline No & 33 & 289 & \\
\hline Vascular invasion & & & 0.256 \\
\hline Yes & 12 & 135 & \\
\hline No & 21 & 155 & \\
\hline Vascular tumor thrombus & & & 0.428 \\
\hline Yes & 9 & 99 & \\
\hline No & 24 & 191 & \\
\hline
\end{tabular}

adenocarcinoma, especially considering the established association between VTE and increased risk of death and early death (2).

Literature review found that older age was considered as a risk factor for lung cancer with VTE (11). Similarly, higher BMI meant higher risk rate of VTE (12), which was consistent with our conclusions. In the analysis of gene test results, the evaluation of whether tumor gene mutation affects the risk of thrombosis has been the focus of preclinical research and clinical research. Our study showed that patients with EGFR mutations had a higher risk of VTE than those with wild-type EGFR (17\% vs. $6 \%, \mathrm{P}=0.002)$. Review of literature data shows that $\mathrm{EGFR}$ 
Table 6 Logistic regression analysis of venous thromboembolism in patients with lung cancer surgery

\begin{tabular}{lcccc}
\hline Variables & $\mathrm{B}$ & $\mathrm{P}$ & $\mathrm{OR}$ & $95 \% \mathrm{Cl}$ \\
\hline BMI & -0.025 & 0.537 & 0.975 & $0.901-1.056$ \\
D-dimer (d1-pre) & 0.238 & 0.014 & 1.269 & $1.050-1.533$ \\
FEV1 & -1.279 & 0.001 & 0.278 & $0.145-0.532$ \\
Surgical procedure & -0.259 & 0.639 & 0.772 & $0.262-2.276$ \\
Length of surgery & 0.001 & 0.814 & 1.001 & $0.993-1.008$ \\
Bleeding & 0.001 & 0.226 & 1.001 & $0.999-1.003$ \\
EGFR mutation & 0.984 & 0.014 & 2.675 & $1.220-5.861$ \\
Acinar & 1.014 & 0.012 & 2.757 & $1.247-6.096$ \\
\hline
\end{tabular}

BMI, body mass index; FEV1, forced expiratory volume-one second.

amplification or mutation can induce excessive expression of TF in cancer cells $(13,14)$. Increased TF may constitute a direct association between thrombosis risk and oncogene expression. We hypothesized that EGFR mutations might increase TF expression, thereby increasing tumor coagulant activity and VTE incidence. However, previous studies have reported inconsistent correlations between $E G F R$ gene status and VTE risk in lung adenocarcinoma patients $(15,16)$. One possible explanation for the different results is that previous studies included patients from different ethnic groups. Limited clinical data indicate that there is no clinical link between the mutation status of KRAS tumor and the systemic hypercoagulability of cancer patients (15). In our study, KRAS mutation was not independently correlated with the risk of VTE in patients with invasive lung adenocarcinoma, which was consistent with some research results. Relevant literature suggested that $A L K$ gene mutation increased the risk of VTE in patients with NSCLC (1), which was not reflected in our study. We consider that it may be related to the selection of sample enrollment and the difference of sample size. The D-dimer level represents the preoperative coagulation state of the patient, which has high sensitivity and poor specificity, and is mostly used for auxiliary diagnosis and prognosis monitoring of VTE (17). Therefore, we compared the changes of D-dimer in patients before and on the first day after the operation, and we believed that the difference between the VTE group and the control group was more significant, and the difference was statistically significant
$(\mathrm{P}=0.001)$. Our results suggest that FEV1 level is an independent risk factor for lung cancer associated with VTE. We speculate that this may be related to vascular endothelial injury caused by hypoxia. Lung cancer surgery involves the blocking of important blood vessels, which will inevitably cause local venous injury, while vascular endothelial injury will expose collagen and basement membrane, release TF, promote platelet agglutination, and lead to thrombosis. In the analysis of surgical related factors, there were statistically significant differences in surgical methods (thoracotomy vs. VATS), surgical duration and blood loss between the VTE group and the control group $(\mathrm{P}<0.05)$. In the analysis of tumor pathological data, the proportion of acinar dominant lung adenocarcinoma in the VTE group was higher than that in the non-VTE group $(57.6 \%$ vs. $30.7 \%, \mathrm{P}=0.002)$. The other types of lung adenocarcinoma showed no statistical difference between the VTE group and the control group. The results of this study may be due to the relatively small sample size, and the included subjects were all patients with invasive lung adenocarcinoma, excluding patients with micro-invasive lung adenocarcinoma and adenocarcinoma in situ. The conclusion may be biased, which needs to be confirmed in further studies. A number of studies have shown that low degree of tumor differentiation and advanced tumor are high risk factors for VTE (18-21), while studies on new classification of lung adenocarcinoma and its correlation with VTE are still lacking. The subjects of this study were patients with invasive lung adenocarcinoma who retained complete pathological and genetic test data after surgery, to determine the incidence of VTE in patients with EGFR mutated lung adenocarcinoma. Compared with other studies on lung cancer complicated with VTE, the conclusion has certain limitations, and more clinical data are needed to support it. As far as we know, our observation of the high VTE rate of acinar primary lung adenocarcinoma in the new classification of lung adenocarcinoma is the first report. Further research needs to be done in a larger prospective cohort.

To sum up, the incidence of VTE was $10.2 \%$ in patients with invasive lung adenocarcinoma without prophylactic anticoagulant therapy. EGFR gene mutation is an independent risk factor for postoperative VTE in lung cancer, and the incidence of VTE in adenocarcinoma with alveolar predominance is the highest. Other independent risk factors included the difference of D-dimer (d1-pre) and 
preoperative FEV1.

\section{Acknowledgments}

None.

\section{Footnote}

Conflicts of Interest: The authors have no conflicts of interest to declare.

Ethical Statement: The authors are accountable for all aspects of the work in ensuring that questions related to the accuracy or integrity of any part of the work are appropriately investigated and resolved. The study was approved by the Ethics Committee of Beijing Chaoyang Hospital (No.2019-D.-221-1). The participants provided written informed consent to participate in this study. The participants provided written informed consent to participate in this study.

\section{References}

1. Song CF, Li H, Tian B, et al. Incidence of postoperative venous thromboembolism after thoracic surgery and its characteristic: a single center, prospective cohort study. Zhonghua Wai Ke Za Zhi 2018:284-8.

2. Zer A, Moskovitz M, Hwang D, et al. OA21.02 ALKRearranged Non-Small Cell Lung Cancer is Associated with a High Rate of Venous Thromboembolism. J Thorac Oncol 2017;12:S134.

3. Hirsch FR, Varella-Garcia M, Bunn PA Jr, et al. Epidermal growth factor receptor in non-small-cell lung carcinomas: correlation between gene copy number and protein expression and impact on prognosis. J Clin Oncol 2003;21:3798-807.

4. Ono M, Kuwano M. Molecular mechanisms of epidermal growth factor receptor (EGFR) activation and response to gefitinib and other EGFR-targeting drugs. Clin Cancer Res 2006;12:7242-51.

5. Yarden Y. The EGFR family and its ligands in human cancer : signalling mechanisms and therapeutic opportunities. Eur J Cancer 2001;37:S3-8.

6. Travis WD, Elisabeth B, Masayuki N, et al. International Association for the Study of Lung Cancer/American Thoracic Society/European Respiratory Society: international multidisciplinary classification of lung adenocarcinoma: executive summary. Proc Am Thorac Soc
2011;8:381-5.

7. Zhang X, Li H, Chen W, et al. Efficacy and safety of chemotherapy for newly diagnosed advanced non-small cell lung cancer with venous thromboembolism. Thorac Cancer 2015;6:772-7.

8. Wang Z, Yan HH, Yang JJ, et al. Venous thromboembolism risk factors in Chinese non-small cell lung cancer patients. Support Care Cancer 2015;23:635-41.

9. Heit JA. Epidemiology of venous thromboembolism. Nat Rev Cardiol 2015;12:464-74.

10. Yang Y, Zhou Z, Niu XM, et al. Clinical analysis of postoperative venous thromboembolism risk factors in lung cancer patients. J Surg Oncol 2012;106:736-41.

11. Lee YG, Kim I, Lee E, et al. Risk factors and prognostic impact of venous thromboembolism in Asian patients with non-small cell lung cancer. Thromb Haemost 2014;111:1112-20.

12. Lindström S, Germain M, Crous-Bou M, et al. Assessing the causal relationship between obesity and venous thromboembolism through a Mendelian Randomization study. Hum Genet 2017;136:897-902.

13. Magnus N, Garnier D, Rak J. Oncogenic epidermal growth factor receptor up-regulates multiple elements of the tissue factor signaling pathway in human glioma cells. Blood 2010;116:815-8.

14. Rong Y, Belozerov VE, Tucker-Burden C, et al. Epidermal growth factor receptor and PTEN modulate tissue factor expression in glioblastoma through JunD/ activator protein-1 transcriptional activity. Cancer Res 2009;69:2540-9.

15. Corrales-Rodriguez L, Soulières D, Weng X, et al. Mutations in NSCLC and their link with lung cancerassociated thrombosis: a case-control study. Thromb Res 2014;133:48-51.

16. Verso M, Chiari R, Mosca S, et al. Incidence of Ct scandetected pulmonary embolism in patients with oncogeneaddicted, advanced lung adenocarcinoma. Thromb Res 2015;136:924-7.

17. Zhang PP, Sun JW, Wang XY, et al. Preoperative plasma $\mathrm{D}$-dimer levels predict survival in patients with operable non-small cell lung cancer independently of venous thromboembolism. Eur J Surg Oncol 2013;39:951-6.

18. Hicks LK, Cheung MC, Ding K, et al. Venous thromboembolism and nonsmall cell lung cancer: a pooled analysis of National Cancer Institute of Canada Clinical Trials Group trials. Cancer 2009;115:5516-25.

19. Shepherd F, Hasan B, Hicks L, et al. 6516 ORAL Venous thromboembolism (VTE) and non-small cell lung cancer 
(NSCLC): a pooled analysis of National Cancer Institute of Canada Clinical Trials Group (NCIC CTG) trials. EJC Suppl 2007;5:361.

20. Sundbøll J, Hováth-Puhó E, Adelborg K, et al. Risk of arterial and venous thromboembolism in patients with atrial fibrillation or flutter: A nationwide population-based cohort study. Int J Cardiol 2017;241:182-7.

21. Vanassche T, Verhamme P, Wells PS, et al. Impact of age, comorbidity, and polypharmacy on the efficacy and safety of edoxaban for the treatment of venous thromboembolism: An analysis of the randomized, doubleblind Hokusai-VTE trial. Thromb Res 2018;162:7-14.

Cite this article as: Wang J, Hu B, Li T, Miao J, Zhang W, Chen S, Sun Y, Cui S, Li H. The EGFR-rearranged adenocarcinoma is associated with a high rate of venous thromboembolism. Ann Transl Med 2019;7(23):724. doi: 10.21037/atm.2019.12.24 